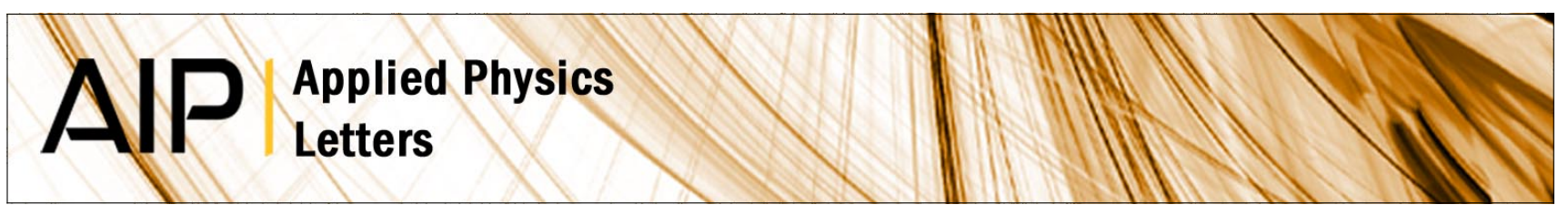

\title{
Observation of $\mathrm{AlGaAs} / \mathrm{GaAs}$ multiquantum well structure by scanning tunneling microscopy
}

J. M. GómezRodríguez, A. M. Baró, J. P. Silveira, M. Vázquez, Y. González et al.

Citation: Appl. Phys. Lett. 56, 36 (1990); doi: 10.1063/1.102639

View online: http://dx.doi.org/10.1063/1.102639

View Table of Contents: http://apl.aip.org/resource/1/APPLAB/v56/i1

Published by the American Institute of Physics.

\section{Related Articles}

Understanding the effect of the layer-to-layer distance on Li-intercalated graphite

J. Appl. Phys. 111, 124325 (2012)

Growth of metal and metal oxide nanowires driven by the stress-induced migration

J. Appl. Phys. 111, 104305 (2012)

Large area Co nanoring arrays fabricated on silicon substrate by anodic aluminum oxide template-assisted electrodeposition

Appl. Phys. Lett. 100, 183101 (2012)

Smallest separation of nanorods from physical vapor deposition

Appl. Phys. Lett. 100, 141605 (2012)

Growth of titanium nanoparticles in confined plasma

Phys. Plasmas 19, 033516 (2012)

\section{Additional information on Appl. Phys. Lett.}

Journal Homepage: http://apl.aip.org/

Journal Information: http://apl.aip.org/about/about_the_journal

Top downloads: http://apl.aip.org/features/most_downloaded

Information for Authors: http://apl.aip.org/authors

\section{ADVERTISEMENT}

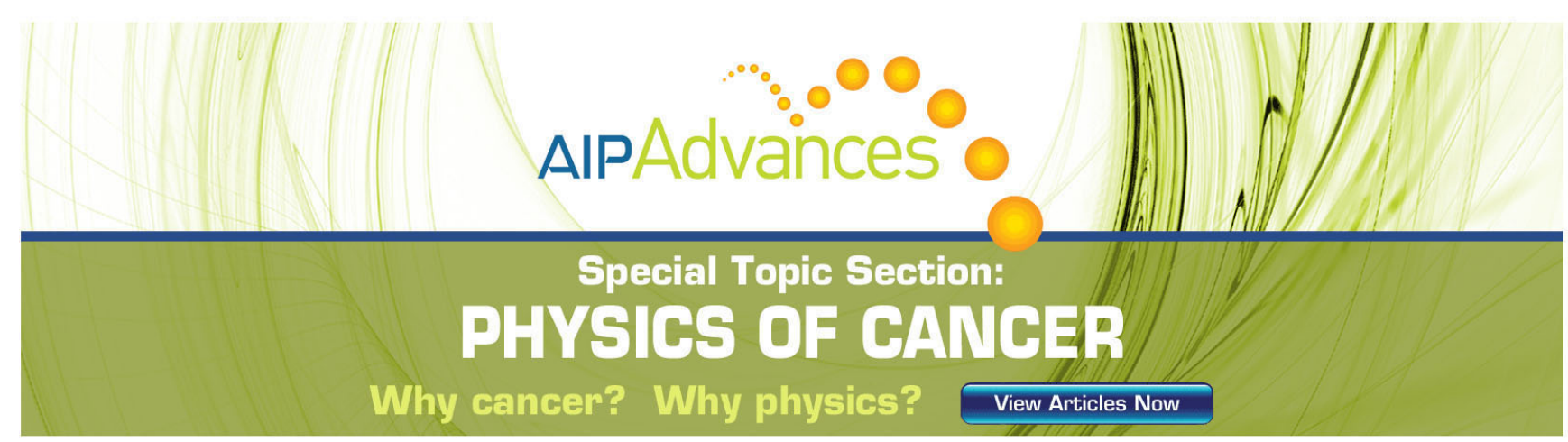




\title{
Observation of AlGaAs/GaAs multiquantum well structure by scanning tunneling microscopy
}

\author{
J. M. Gomez-Rodríguez and A. M. Baro \\ Departamento de Fisica de la Materia Condensada, C-III Universidad Autónoma de Madrid, Cantoblanco, \\ 28049-Madrid, Spain
}

\author{
J.P. Silveira, M. Vázquez, Y. Gonzólez, and F. Briones \\ Centro Nacional de Microelectrónica, Consejo Superior de Investigaciones Científicas, Serrano 144 , \\ 28006-Madrid, Spain
}

(Received 5 July 1989; accepted for publication 30 October 1989)

\begin{abstract}
We have imaged an AlGaAs/GaAs mutiquantum well structure by scanning tunneling microscopy (STM). In order to localize the structure the STM is integrated in a conventional scanning electron microscope. The observed surface structure has a periodicity of $\approx 180 \AA$ and shows an apparent corrugation of $\approx 10 \AA$ in the constant current mode. We discuss the possible mechanisms of the observed contrast, which we tentatively attribute to the different clectrical properties of the two different layers.
\end{abstract}

Recently scanning lunneling microscopy (STM) has been extensively used to determine the surface structure at atomic resolution of metals and semiconductors.' STM has aiso been applied in the nanometer range to study the structure of microfabricated features which are so important in the development towards a higher integration in electronic circuits. Data on GaAs-nanofabricated dots have been recently presented. ${ }^{2}$ STM can be very useful for evaluation of semiconductor device fabrication processes, in particular epitaxial growth of heterostructures and superlattices, by direct observation of a cieaved cross section of the structure with nanometer resolution. No complicated sample preparation is needed and contrast between different materials is excellent.

In this letter we present a study by STM on a multiquantum well (MQW) structure of $A 1 G a A s / G a A s$. We are able to precisely localize the MQW layers by using a combined scamning tunneling microscopy/scanning electron microscopy (STM/SEM) instrument developed in our laboratory. ${ }^{3}$ The images obtained are consistent with the periodicity and the possible origin of the observed contrast is discussed. We compare the data in relation with a previous paper reported by Osaka $a{ }^{4}$ on a different MQW structure.

The tunneing microscony measurements were all performed with a STM placed on the sample stage of a commercial SEM. The experimental setup of our STM/SEM combination has been described in detail elsewhere." The STM piczoelectric tripod was calibrated by imaging the hexagonal lattice of highly oriented pyrolytic graphite at atomic resolution and by measuring a GaAs-nanostructured sample with well-defned parameters. ${ }^{2}$ Nevertheloss, and due to the effect of nonlinearities in the piezoelectric displacement, we cannot give a precision better than $10 \%$.

STM data were acquired by means of a PC-AT data acquisition and image processing system. The scan rates ranged from 1 to $3 \mathrm{~Hz}$. Barrier height measarements were made following a well established technique.

The sample is a unifomly Si-doped $\left(n=1.5 \times 10^{18}\right.$ $\left.\mathrm{cm}^{-3}\right) \mathrm{Al}_{0.3} \mathrm{Ga}_{0.7} \mathrm{As} / \mathrm{GaAs}$ miltiquantum well structure grown by atomic layer molecular beam epitaxy $(\mathrm{ALMBE})^{5}$ at $400^{\circ} \mathrm{C}$, on Si-coped $\left(n=1.2 \times 10^{18} \mathrm{~cm}^{-3}\right)$ GaAs $(001)$ substratc. Post-growth x-ray characterization of the structure gives a MQW periodicity of $186 \AA$, with 124 and $62 \AA$ the respective thicknesses for the individual layers of GaAs and $\mathrm{Al}_{0.3} \mathrm{Ga}_{0.7} \mathrm{As}$, in good agreement with nominal growth parameters determined by reflection high-energy electron diffaction (RHEED). The total thickness is $0.6 \mu \mathrm{m}$. Interface sharpness is expected to be very good by ALMBE and interface roughness imited to less than one monolayer.

The sampie was cleaved in air and then quickly transferred to the STM/SEM vacuum chamber; the base pressure was $\approx 10^{-6}$ mbar during all the measurements. Figure 1 (a) schematically illustrates the geometry of the sample with respect to the STM tip. Figure 1 (b) is a SEM micrograph showing, at very low magnification, the position of the tip on the very edge of the freshly cleaved sample.

In Fig. 2 we display the STM data measured in the same region as Fig. 1(b). Figure 2 (a) is a shaded top view with a simulated light source located on the right-hand side of the image. Figure $2(b)$ is a three-dimensional shaded view of the same data. Tumnel current was fixed to $3.0 \mathrm{nA}$ and tip-tosample voltage was $3.3 \mathrm{~V}$ (tip positive).

As measured in Fig. 2(a), the periodicity of the MQW structure is $180 \AA+10 \%$. This periodicity agrees, within the accuracy of the piezcelectric calbration, with the sum of the thickness of one GaAs layer (124 A) and one AlGaAs $(62 \AA)$ layer.

The most important point to discuss is the origin of the observed apparent corrugation. If we take into account the basic expression relating tunneling voltage $V$, current $l$, gap $s$, and barrier height $\phi$

$$
I=f(V) \exp \left(-\phi^{1 / 2} s\right) \text {, }
$$

we see that the three sources of contrast are morphology $s$, chemical composition $\phi$, and electrical properties $f(V)$. In the following we analyze these three factors.

The data show that some morphological efects exist. This can be seen by looking at the profile of the structure where two well-marked corrugation jumps of $\approx 30 \AA$ height are visible. We believe that these features are true steps produced during the cleavage process. The fact that the steps appear just along the interface can be understood because 


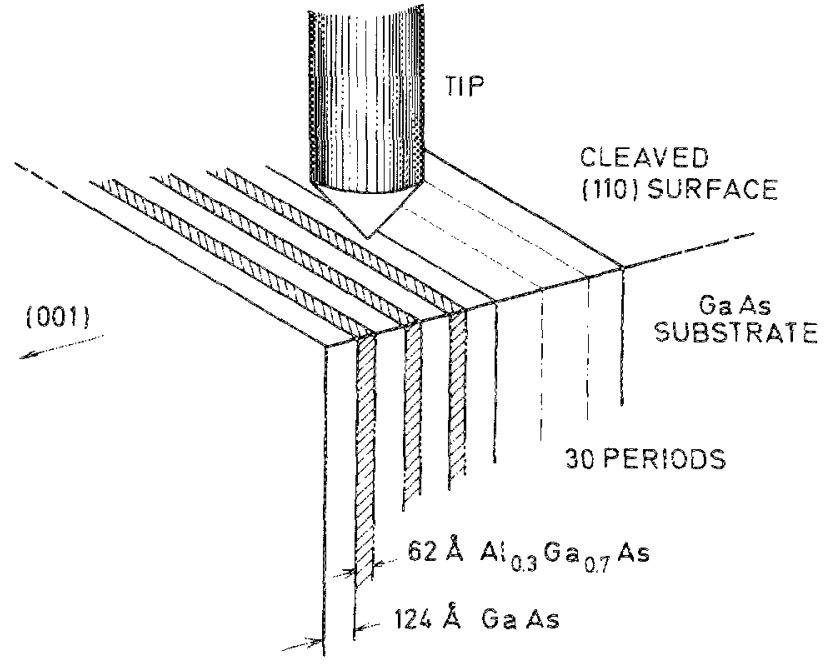

(a)

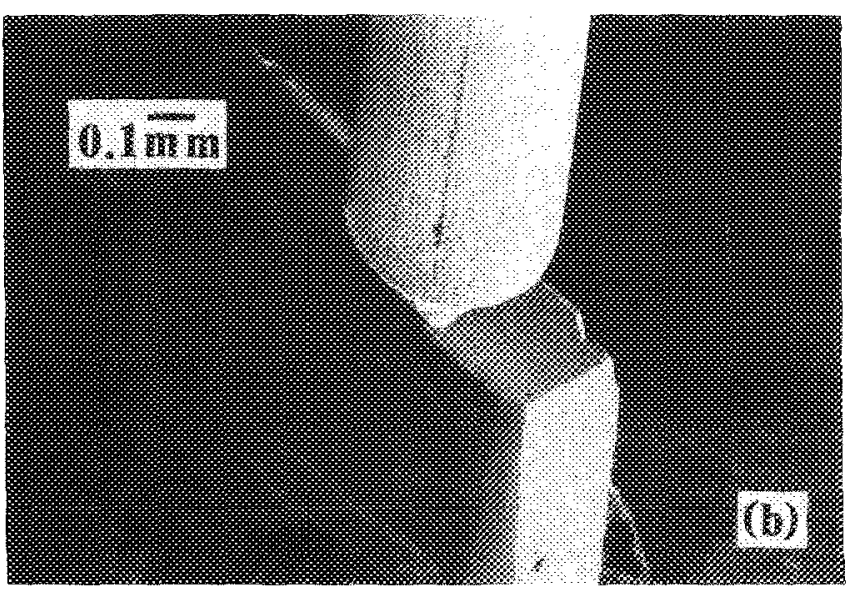

MG. 1. (a) Schematic drawing of the MOW structure, with the periodicity of the AlGaAs and GaAs layers obtained by $\mathrm{x}$-ray characterization. (b) Low magnification SEM micrograph showing the position of the STM tip on the very edge of the freshly cleaved sample.

the interface provides a privileged place to release the stress produced during the cleavage process. We have also found bunches of steps on the cleaved surface of the substrate. The image also shows a less pronounced step which crosses the structure from top keft to bottom right. In this case, the morphological contrast occurs within exch layer.

In addition to the above-mentioned features which we attribute to steps, there is a uniform contrast between the layers of AlGaAs and GaAs equivalent to a corrugation of $\approx 10 \mathrm{~A}$. Such a contrast allows us to distinguish between the layers. According to the data about the perfection of the interface, we do not think that this contrast is due to morphological effects.

An obvious source of contrast is the change in chemical composition between the two layers. This could be reflected in the value of the barrier height. We have measured its actual value together with the topography by the modulation technicue, obtaining an average of $0.2 \mathrm{eV}$. Although this value is anomalously low, it is the typical one obtained by STM on samples which are not clean (due to the vacuum not better than $10^{-6} \mathrm{mbar}$ ). This low value is not different from the one obtained on metals under the same vacuum condi-
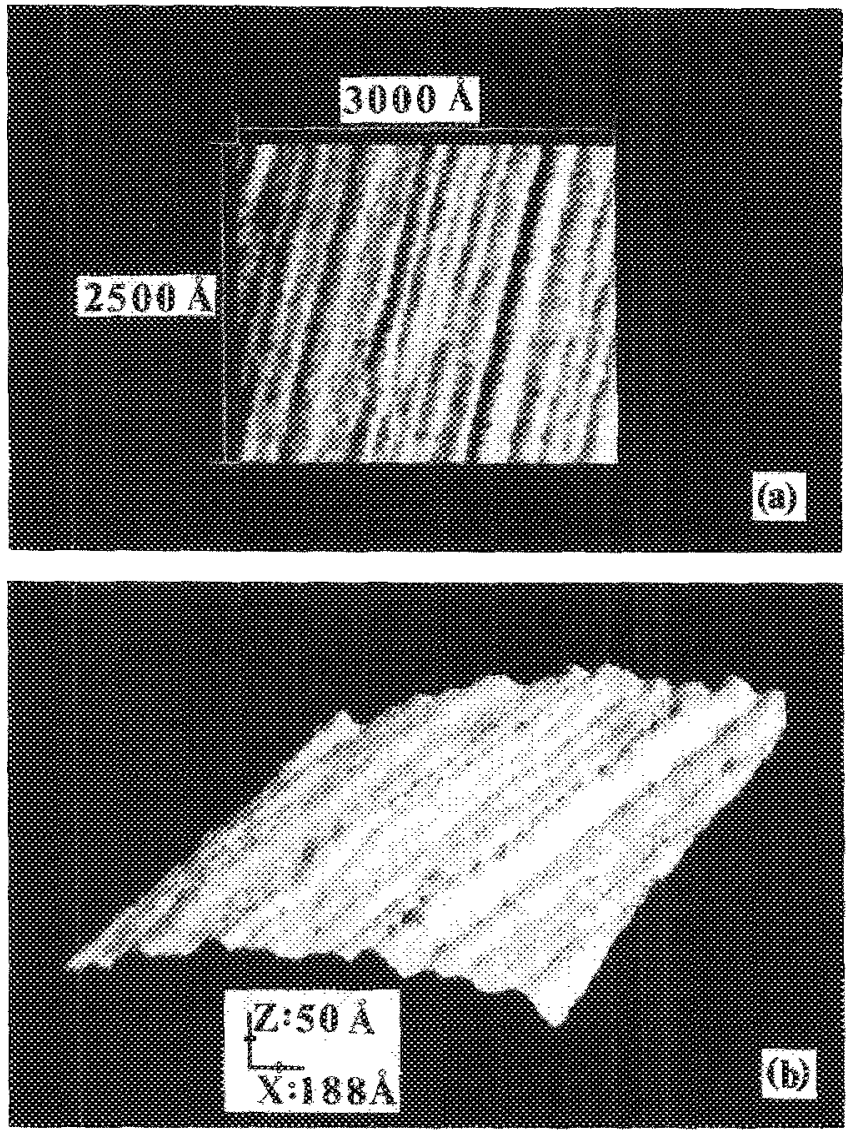

FIG. 2. STM data measured on the sample shown in Fig. 1. Tip voltage was $+3.3 \mathrm{~V}$ and tunnel current was $3 \mathrm{nA}$. Scanning frequency in the $x$ direction was $2 \mathrm{~Hz}$. (a) Shaded top vicw with a simulated light source located on the right-hand side of the inage at $35^{\circ}$ above the $X Y$ plane. (b) Three-dimensional shaded view of the same data.

tions. The reason for this behavior is not yet completely understeod. Besides this theoretical problem, our experiments could not clearly identify a contrast between the layers in the measurement of $\phi$.

Let us now finaly discuss the electrical properties. There are two factors which must be considered:

(i) The band-gap value. As it is known, the band gap varies from 1.4 to $1.8 \mathrm{eV}$ when passing from GaAs to AlGaAs." As a result of that, when tunneling from the valence band, i.e., for sample tunncling voltage negative, the 1 $V$ curves shift to higher voltages in the AlGaAs layer. ${ }^{7}$ Thus, as the experment is done at $V$ and $I$ constant, the tip will be closer to the sample when entering the AlGaAs layer.

(ii) The voliage drop. Due to the tunneling current, a charge depletion in the surface region produces a band bending. This voltage drop depends on the equipotential lines inside the material in the spreading resistance region. ${ }^{8}$ The large needed value of $V(3.3 \mathrm{~V})$ suggests that such a voltage drop actually exists in our case. Its value mighe be different in the two materials due to differential oxidation and/or differential adsorption of oxygen. Oxidation of the GaAs (110) surface is well documented. Bartels et al. ${ }^{\prime}$ report that after $10^{14} \mathrm{~L}$ of oxygen exposure, the oxide film formed on GaAs consists of two layers. On AlGaAs the data are almost nonexistent, although a much higher reactivity is expected. Sec- 
ondary-ion mass spectrometry (SIMS) measurements by Amano et al. ${ }^{10}$ on a molecular beam epitaxy (MBE) grown AIGaAs/GaAs heterostructure, show that the oxygen content on AlGaAs is more than ten times higher than in GaAs. $A$ difference in the amount of oxidation will produce a change in tunneling conductance on the two materials. We cannot quantify, however, its actual infuence aithough it would also lead to a minimum on AlGaAs.

Any of these processes will change the pre-exponential factor $f(V)$ to be used in expression (1). A change in $f(V)$, even if small, has a big infuence on $s$, because of the low value of $\phi$. For example, ising the experimental value of $\phi=0.2 \mathrm{eV}$, a change in $f(V)$ by a factor of 2 produces a jump in $s$ of $10 \AA$.

Although we cannot definitely decide between the varlous sources of contrast discussed before, we believe that the last point connected with the effect of electrical properties is the more reasonable explanation to our data. This argument attributes a minimum to AlGaAs and a maximum to GaAs.

We inally discuss the previous data reported by Osaka et $a l^{4}$ These authors atribute the contrast to the band-gap value. The corrugation, however, is higher than in our case, reaching $150 \AA$ at a tunneling voltage of $0.5 \mathrm{~V}$. Such corrugation values are very high and diffeult to understard, as the authors recognize. In order to test the relationship between STM corrugation and real topography, they evaporated a Au film on the cleaved surface. They conclude from this experiment that STM corrugation does not come from the topography of the MQW structure. In our case a similar test cannot be successful as we get much lower corrugation.

In summary, we have measured a MOW structure of AlGaAs/GaAs by means of a \$TM/SEM combined instrument. The observed corrugation is tentatively attributed to a change in the electrical properties of the two different layers.

The author are very especially indebted to José Luis De Miguel. Without his interest, encouragement, and help in the choice and preparation of samples as well as in the discussion of preliminary results this work would not have been possible. They also thank J. Gómez-Herrero and L. Vazquez. for discussions. This work has been financially supported by the CICyT under contract No. PB86-0606.

'G. Binnig and H. Kohrer, IBM J. Res. Develop. 30, 355 (1986).

${ }^{2} J$. M. Gómez-kodríguez, L. Vázquez., A. Bartolomés A. M. Baró, P. Grambow, and D. Ileitmann, Utramicroscopy 30, 355 (1989).

3. Vazcuez, A. Bartolomé, R. Garcia, A. Buendía, and A. M. Baró, Rev. Sci. Instrum. 59, $1286(1988)$.

4F. Osaka, I. Tanaka, T. Kato, and Y. Katayama, Jpn. J. Appl. Phys. 27, $1193(1988)$.

'A. Ruiz, L. Gonzalez, A. Mazuelas, and F. Briones, Appl. Phys. A (in press).

"H. C. Casey, Jr. and M. B. Panish, Heterostructure Lasers (Academic, New York, 1978), Parts A and $B$.

${ }^{\prime}$ H. W. M. Salemink, H. P. Meier, R. Elliatiogiu, J. W. Gerritsen, and P. R. M. Muralt, Appl. Phys. Lett. 54, 1112 (1989).

${ }^{7}$ F. Flores and N. García, Phys. Rev. B 30, 2289 (1984).

"F. Bartels, H. Groll, and W. Mönch, Solid State Commun. 57, 571 (1986).

:Ch. Amano, K. Ando, and M. Yamaguchi, J. Appl. Phys. 63, 2853 (1988). 